\title{
Sparse Polynomial-Chaos Models for Stochastic Problems with Filtering Structures
}

\author{
Theodoros Zygiridis $^{1 *}$, Aristeides Papadopoulos ${ }^{2}$, Nikolaos Kantartzis ${ }^{3}$, and Elias Glytsis ${ }^{2}$ \\ ${ }^{1}$ Department of Electrical and Computer Engineering, University of Western Macedonia, Kozani, Greece \\ ${ }^{2}$ School of Electrical and Computer Engineering, National Technical University of Athens, Athens, Greece \\ ${ }^{3}$ Department of Electrical and Computer Engineering, Aristotle University of Thessaloniki, Thessaloniki, Greece \\ *corresponding author, E-mail: tzygiridis@uowm.gr
}

\begin{abstract}
This work develops sparse polynomial models for investigating the response of electromagnetic filtering structures, when the design of the latter is affected by a number of uncertain variables. The proposed approach describes an improved implementation framework for contemporary Compressed Sensing techniques, which are known for their capacity to reconstruct sparse signals with a limited number of samples. Unlike typical implementations, the required set of basis functions is formulated after performing an initial estimation of partial variances that, despite being computationally cheap, provides sufficient information for the impact of each variable on the output. A number of numerical tests on different filter configurations verify the reliability of the presented methodology, display its efficiency, and unveil the performance of the considered structures, when operated under uncertainty.
\end{abstract}

\section{Introduction}

Electromagnetic (EM) filtering structures appear in numerous classic and modern applications spanning diverse parts of the spectrum, including the radio- [1], microwave$[2,3]$, millimeter- [4], THz- [5] and optical- [6,7] frequency bands, to name a few representative instances. In most cases, the consistent operation of such configurations requires the compliance with specific - and, at the same time, probably firm - design guidelines, and deviations from the latter are likely to cause undesirable performance degradation or even failures. Consequently, the ability to predict the response of filtering structures under uncertainty conditions appears to be a matter of importance, due to the necessity for assessing key features, such as their functionality sensitivity on potential manufacturing flaws.

Given the aforementioned framework, the computation of generalized polynomial-chaos (PC) expansions [8] of stochastic quantities of interest (QoIs) is considered an attractive solution, as these are used extensively in the construction of surrogate models for problems with uncertainties. When a QoI depends on a small or moderate number of random variables, PC approaches are proven to perform more efficiently than traditional methodologies, among which Monte-Carlo (MC) techniques [9] constitute common choices. On the other hand, these high-performance levels of the PC expansions are not always guaranteed and their computation may require more advanced approaches, in case of several random inputs or expensive deterministic models. This stems from the fact that the computational cost for the determination of the pertinent expansion coefficients is directly related to the number of basis functions incorporated in the PC formulae. In general, finding efficient solutions for multi-dimensional stochastic problems is a non-trivial, but of crucial importance, issue and a number of different solutions have been proposed, including sparse grids [10], alternative construction methodologies for the basis sets [11], adaptive schemes [12], etc.

Regarding the development of sparse PC models (i.e. with a low number of non-zero terms), Compressed Sensing (CS) is known to be more cost-effective than standard methodologies (e.g. least-squares techniques), provided that the PC coefficients form a sufficiently sparse vector [13]. The efficiency of CS stems from the fact that the reconstruction of sparse vectors is possible using a limited number of samples, which can be smaller than the number of unknowns. As regression methodologies commonly require increased amounts of samples (two to three times the number of unknowns) for the formulation of an overdetermined system, one easily recognizes the advantages that CS has to offer. These capabilities have been recently exploited with success in the context of PC methodologies [14-16], and will be also used extensively in the present work.

Due to constantly increasing interest, ongoing research efforts continue to produce novel or improved solutions for challenging problems with uncertainties. For instance, various modern approaches for stochastic problems with several dimensions are presented in [17]. These methodologies include collocation schemes that exploit Leja sequences with dimension-adaptive characteristics, the construction of PC models with two levels of adaptivity, and the cost-effective computation of statistical moments via lowrank tensor decompositions. In cases with random input variables of unknown type, [18] suggests the approximation of the output probability-density function (PDF) using the generalized lambda distribution. The involved parameters are related to the inputs via PC expansions, and accurate surrogate models are developed using optimization- 
entailing algorithms. Regarding the sensitivity analysis of time-dependent stochastic systems, [19] calculates the Sobol indices over extended time periods, rather than in a pointwise fashion, so that the least important input variables are identified reliably and set to their deterministic nominal values. In this context, surrogate modelling via PC expansions and utilization of either spectral projection or CS may provide a sufficient framework for computing the Sobol indices. The possibility of consistently reconstructing sparse signals using fewer samples than standard methodologies is demonstrated in [20], by learning the measurement matrix in a data-driven fashion that identifies structures other than sparsity in the unknown vectors. A mixed $\ell_{1}-\ell_{2}$ regularization technique is used in [21] for the computation of the PC coefficients, combined with a re-ordering procedure for the basis function that aims to select the elements of the basis set with the highest PC coefficients. Numerical results reveal improved convergence rates, compared to typical sparse methods, especially for problems with high dimensionality.

In this paper, we adopt a CS approach for the study of stochastic problems involving filters, considering that their responses are commonly affected by several EM or geometric factors. We propose the incorporation of an extra stage before the computation of the PC expansion coefficients, within which an approximate variance analysis can be conducted in a cost-effective fashion. Thus, an initial estimation of the importance of each input is formed, according to which unnecessary basis functions may be dropped without accuracy compromise, improving eventually the overall efficiency. Until today, only a limited number of works have given attention to the truncation scheme of the PC expansions, in the context of CS techniques (for instance, [16] develops an adaptive basis-selection methodology that can be used in conjunction with $\ell_{1}$-minimization). A number of numerical tests display the reliability of the suggested approach for the development of sparse PC models, when filtering configurations operate under uncertain conditions, and useful statistics characterizing their stochastic behavior are computed.

\section{Methodology}

\subsection{Polynomial-Chaos Expansions}

Suppose that the QoI $y$ is a function of $d$ independent random variables $\boldsymbol{\xi}=\left[\begin{array}{lll}\xi_{1} & \ldots \xi_{d}\end{array}\right]^{\mathrm{T}}$ of known type, as well as of some deterministic variables represented by $\zeta$ (e.g. frequency). A truncated PC representation of $y$ has the form

$$
y(\boldsymbol{\zeta} ; \boldsymbol{\xi}) \simeq \hat{y}(\boldsymbol{\zeta} ; \boldsymbol{\xi})=\sum_{\boldsymbol{\alpha} \in \mathcal{A}} c_{\boldsymbol{\alpha}}(\boldsymbol{\zeta}) \Psi_{\boldsymbol{\alpha}}(\boldsymbol{\xi})
$$

where $\boldsymbol{\alpha}=\left(\alpha_{1}, \ldots, \alpha_{d}\right) \in \mathbb{N}_{0}^{d}$ is a multi-index, $c_{\boldsymbol{\alpha}}$ are the expansion coefficients, $\Psi_{\alpha}$ are the basis functions, and $\mathcal{A}$ is the set of basis indices [8]. The basis functions are constructed via

$$
\Psi_{\boldsymbol{\alpha}}(\boldsymbol{\xi})=\prod_{i=1}^{d} \psi_{\alpha_{i}}\left(\xi_{i}\right)
$$

where $\psi_{\alpha_{i}}$ is a univariate polynomial of degree $\alpha_{i}$, selected according to the Askey scheme [8], which matches the basis functions with the distribution of the input variables for optimum performance. In this work, we consider uniformly distributed random variables, and the suitable choice in this case corresponds to Legendre polynomials. The basis functions are orthogonal with respect to the joint PDF,

$$
f(\boldsymbol{\xi})=\prod_{i=1}^{d} f_{i}\left(\xi_{i}\right)
$$

where $f_{i}\left(\xi_{i}\right)$ is the PDF of the $i$-th variable. Consequently, it is $\left\langle\Psi_{\boldsymbol{\alpha}_{i}}(\boldsymbol{\xi}), \Psi_{\boldsymbol{\alpha}_{j}}(\boldsymbol{\xi})\right\rangle=\left\|\Psi_{\boldsymbol{\alpha}_{i}}(\boldsymbol{\xi})\right\|^{2} \delta_{\boldsymbol{\alpha}_{i} \boldsymbol{\alpha}_{j}}$, where

$$
\left\langle\Psi_{\boldsymbol{\alpha}_{i}}(\boldsymbol{\xi}), \Psi_{\boldsymbol{\alpha}_{j}}(\boldsymbol{\xi})\right\rangle=\int_{\Omega} \Psi_{\boldsymbol{\alpha}_{i}}(\boldsymbol{\xi}) \Psi_{\boldsymbol{\alpha}_{j}}(\boldsymbol{\xi}) f(\boldsymbol{\xi}) d \boldsymbol{\xi}
$$

$\left\|\Psi_{\boldsymbol{\alpha}_{i}}(\boldsymbol{\xi})\right\|^{2}=\left\langle\Psi_{\boldsymbol{\alpha}_{i}}(\boldsymbol{\xi}), \Psi_{\boldsymbol{\alpha}_{i}}(\boldsymbol{\xi})\right\rangle, \delta_{\boldsymbol{\alpha}_{i} \boldsymbol{\alpha}_{j}}=1$ if $\boldsymbol{\alpha}_{i}=\boldsymbol{\alpha}_{j}$ and $\delta_{\boldsymbol{\alpha}_{i} \boldsymbol{\alpha}_{j}}=0$ in any other case, and $\Omega$ is the $d$-dimensional random space.

Furthermore, $\mathcal{A}$ is constructed according to a certain truncation rule. For example, a general and widely used choice is described by

$$
\mathcal{A}_{q}=\left\{\boldsymbol{\alpha} \in \mathbb{N}_{0}^{d}:\|\boldsymbol{\alpha}\|_{q} \leq p\right\}
$$

where $p$ is the selected polynomial order and

$$
\|\boldsymbol{\alpha}\|_{q}=\left(\alpha_{1}^{q}+\ldots \alpha_{d}^{q}\right)^{1 / q}
$$

with $q \leq 1$. The cardinality $N+1$ of $\mathcal{A}_{1}(q=1)$ in (5), which corresponds to the total-degree rule, is

$$
N+1=\frac{(p+d) !}{p ! d !}
$$

and attains large values in case of stochastic problems with several dimensions. The reduction of the number of basis function is possible by resorting to hyperbolic indexing [11], which amounts to selecting $q<1$ in (5). In this way, functions describing complex contributions are excluded from the basis set. Furthermore, anisotropic index sets may be constructed by enforcing a different maximum polynomial order $\left(p_{1}, \ldots, p_{d}\right)$ for each variable. Such an approach can be proven useful, when information about the impact of each input on the QoI is already available [22]. It is noted that the anisotropic and hyperbolic truncation rules can be easily combined and adapted to a problem's specific requirements. Some representative examples of the aforementioned truncation schemes when $d=3$ can be found in Fig. 1.

Finally, the availability of a PC expansion enables the direct computation of fundamental statistics, such as the expected value and the variance of $y$, according to

$$
\begin{gathered}
\sigma^{2}\{y\}=\sum_{\boldsymbol{\alpha} \in \mathcal{A} \backslash\left\{\boldsymbol{\alpha}_{0}\right\}}^{\mathrm{E}\{y\}=c_{\boldsymbol{\alpha}_{0}}} c_{\boldsymbol{\alpha}}^{2}(\boldsymbol{\zeta})\left\|\Psi_{\boldsymbol{\alpha}}(\boldsymbol{\xi})\right\|^{2}
\end{gathered}
$$

respectively, where $\boldsymbol{\alpha}_{0}=(0,0, \ldots, 0)$. 


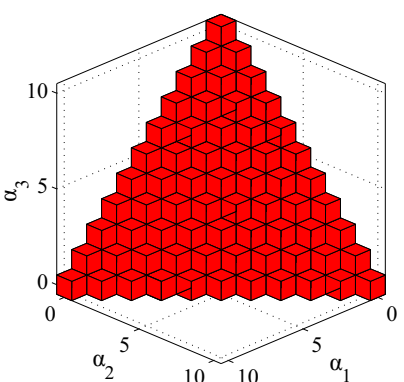

(a)

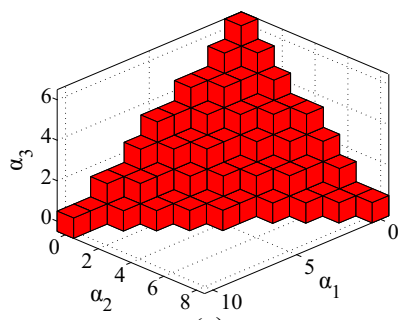

(c)

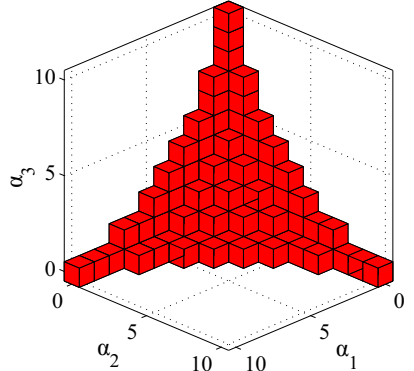

(b)

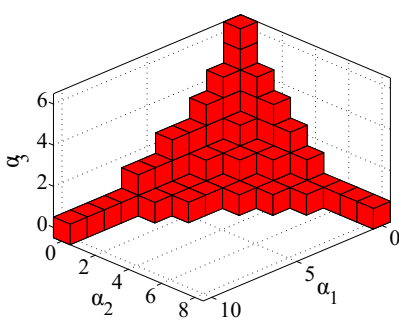

(d)
Figure 1: Index sets for different truncation schemes when $d=3$ : (a) isotropic truncation with $p=10$ and $q=1$ (286 functions), (b) isotropic truncation with $p=10$ and $q=0.7$ (120 functions), (c) anisotropic truncation with $p_{1}=10, p_{2}=8, p_{3}=6$ and $q=1$ (139 functions), and (d) anisotropic truncation with $p_{1}=10, p_{2}=8, p_{3}=6$ and $q=0.7$ (72 functions).

\subsection{Compressed Sensing}

The computation of the expansion coefficients appearing in a PC series necessitates the availability of a number of deterministic samples $y_{i}, i=1, \ldots, N_{t}$, for which an equal number of input vectors $\boldsymbol{\xi}^{(i)}$ needs to be selected. Using (1), a system of the form

$$
\Psi \mathrm{c}=\mathrm{y}
$$

is derived, where

$$
\boldsymbol{\Psi}=\left[\begin{array}{cccc}
\Psi_{\boldsymbol{\alpha}_{0}}\left(\boldsymbol{\xi}^{(1)}\right) & \Psi_{\boldsymbol{\alpha}_{1}}\left(\boldsymbol{\xi}^{(1)}\right) & \ldots & \Psi_{\boldsymbol{\alpha}_{N}}\left(\boldsymbol{\xi}^{(1)}\right) \\
\Psi_{\boldsymbol{\alpha}_{0}}\left(\boldsymbol{\xi}^{(2)}\right) & \Psi_{\boldsymbol{\alpha}_{1}}\left(\boldsymbol{\xi}^{(2)}\right) & \ldots & \Psi_{\boldsymbol{\alpha}_{N}}\left(\boldsymbol{\xi}^{(2)}\right) \\
\vdots & \vdots & \ddots & \vdots \\
\Psi_{\boldsymbol{\alpha}_{0}}\left(\boldsymbol{\xi}^{\left(N_{t}\right)}\right) & \Psi_{\boldsymbol{\alpha}_{1}}\left(\boldsymbol{\xi}^{\left(N_{t}\right)}\right) & \ldots & \Psi_{\boldsymbol{\alpha}_{N}}\left(\boldsymbol{\xi}^{\left(N_{t}\right)}\right)
\end{array}\right]
$$

c is the vector of expansion coefficients, $\boldsymbol{\alpha}_{0}, \ldots, \boldsymbol{\alpha}_{N}$ are the $N+1$ elements of $\mathcal{A}$, and $\mathbf{y}=\left[y\left(\boldsymbol{\zeta} ; \boldsymbol{\xi}^{(1)}\right) y\left(\boldsymbol{\zeta} ; \boldsymbol{\xi}^{(2)}\right) \ldots y\left(\boldsymbol{\zeta} ; \boldsymbol{\xi}^{\left(N_{t}\right)}\right)\right]^{\mathrm{T}}$ is the vector of the output samples. In a standard line of work, the system (10) is overdetermined by selecting $N_{t}=(2 \sim 3) \times(N+1)$, and a typical least-squares approach can be applied. Evidently, the computational cost involved in the construction of $\mathbf{y}$ may become undesirably large, in case of several random dimensions and/or computationally expensive deterministic models. On the other hand, CS can be selected if under-determined systems $\left(N_{t}<N+1\right)$ are considered, as long as the vector $\mathbf{c}$ is sparse (i.e. only a few elements are non-zero). Naturally, PC expansions are rarely sparse, yet they feature expansion coefficients with rapidly decaying magnitude in most cases. Thus, the information regarding the stochastic properties of a QoI is concentrated in a limited number of terms, and a sparse formula can be deemed sufficient.

Specifically, CS approaches determine the PC coefficients by solving the $\ell_{0}$-minimization problem

$$
\hat{\mathbf{c}}=\underset{\mathbf{c}}{\arg \min }\|\mathbf{c}\|_{0} \quad \text { subject to } \quad \mathbf{\Psi} \mathbf{c}=\mathbf{y}
$$

where $\|\cdot\|_{0}$ is the number of non-zero elements contained in the argument. Given that (12) is an NP-hard problem, a direct solution is not realistic. On the other hand, an approximate solution can be found with the Orthogonal Matching Pursuit (OMP), which is a greedy algorithm. OMP features an iterative procedure, according to which a set of active-column indices $\mathcal{B}^{(i)}$ (initially, $\mathcal{B}^{(0)}=\emptyset$ ) is enriched after the $i$-th iteration has been performed, until the required number of non-zero coefficients is reached, or when the residual $\mathbf{r}^{(i)}$ satisfies a specific criterion (initially, $\mathbf{r}^{(0)}=\mathbf{y}$ ). In more detail, the column $\mathbf{v}$ of $\boldsymbol{\Psi}$ that is most correlated with the current residual, excluding those columns whose indices are already in $\mathcal{B}^{(i-1)}$, is selected at each iteration. The index $j^{*}$ of the most suitable column is identified via

$$
j^{*}=\underset{j \notin \mathcal{B}^{(i-1)}}{\arg \max } \frac{\boldsymbol{\Psi}_{j}^{\mathrm{T}} \mathbf{r}^{(i-1)}}{\left\|\boldsymbol{\Psi}_{j}\right\|_{2}}
$$

and the active column-index set is updated by adding the aforementioned element:

$$
\mathcal{B}^{(i)}=\mathcal{B}^{(i-1)} \cup\left\{j^{*}\right\}
$$

The coefficients are updated via

$$
\mathbf{c}^{(i)}=\arg \min \left\|\mathbf{y}-\mathbf{\Psi}^{(i)} \mathbf{c}\right\|_{2}
$$

where $\boldsymbol{\Psi}^{(i)}$ comprises only the columns of $\boldsymbol{\Psi}$ described by $\mathcal{B}^{(i)}$. The solution of (15) is computed via least-squares. In the final step of the iterative procedure, the residual is updated:

$$
\mathbf{r}^{(i)}=\mathbf{y}-\boldsymbol{\Psi}^{(i)} \mathbf{c}^{(i)}
$$

As several studies have shown, the most significant features of OMP are its simplicity and rapid implementation.

Alternatively, the $\ell_{1}$-minimization problem can be solved,

$$
\hat{\mathbf{c}}=\underset{\mathbf{c}}{\arg \min }\|\mathbf{c}\|_{1} \quad \text { subject to } \quad \mathbf{\Psi} \mathbf{c}=\mathbf{y}
$$

where $\|\boldsymbol{\alpha}\|_{1}=\sum_{i=1}^{d}\left|\alpha_{i}\right|$ (this problem is commonly referred to as Basis Pursuit). In case of non-exact representations, a non-zero error threshold $\epsilon$ can be defined (in order to avoid the strict condition $\mathbf{\Psi} \mathbf{c}=\mathbf{y}$ ) and the minimization problem is formulated as

$$
\hat{\mathbf{c}}=\underset{\mathbf{c}}{\arg \min }\|\mathbf{c}\|_{1} \quad \text { subject to } \quad\|\mathbf{\Psi} \mathbf{c}-\mathbf{y}\|_{2} \leq \epsilon
$$


This is known as Basis Pursuit Denoising. In the present study, we use the SPGL1 package [23] for performing $\ell_{1}$ minimization, which is based on the Spectral Projected Gradient algorithm [24], and has been already utilized and tested in several pertinent publications $[14,15,25]$.

\subsection{Basis Selection via Variance Analysis}

Typical PC approaches involve the a priori selection of the basis set, according e.g. to the total-degree rule or a hyperbolic truncation scheme. Instead of choosing the basis functions in this manner, we propose performing a preliminary screening of the input variables, so that their potential impact on the QoI is assessed, at least in an approximate fashion. Towards this goal, one may consider computing the first-order Sobol indices $S_{i}, i=1, \ldots, d$, as proposed in other similar strategies [26], before proceeding to the final calculation of the PC expansion. It is reminded that the Sobol indices constitute a standard measure for global sensitivity analysis. If a PC representation of the QoI $y$ is available, then the Sobol index that corresponds to the $i$-th variable is computed via

$$
S_{i}=\frac{\sum_{\boldsymbol{\alpha} \in \mathcal{A}_{i}} c_{\boldsymbol{\alpha}}^{2}\left\|\Psi_{\boldsymbol{\alpha}}\right\|^{2}}{\sigma^{2}\{y\}}
$$

where $\mathcal{A}_{i}=\left\{\boldsymbol{\alpha} \in \mathcal{A}: \alpha_{i} \neq 0, \alpha_{j \neq i}=0\right\}$. The numerator in (19) corresponds to the conditional variance due to the $\xi_{i}$ variable only, without taking into account combined interactions of $\xi_{i}$ with other variables. Evidently, computing the variance in the denominator of (19) requires the availability of all but one PC coefficients. To enable a more practical solution, we assume a sparse initial PC approximation that comprises only univariate polynomials:

$$
\mathcal{A}^{*}=\left\{\boldsymbol{\alpha} \in \mathbb{N}_{0}^{d}:\|\boldsymbol{\alpha}\|_{0} \leq 1, \alpha_{i} \leq p\right\}
$$

where the cardinality of $\mathcal{A}^{*}$ is $(p \times d+1)$, which is quite small compared to (7). As we need the $S_{i}$ values just for screening purposes, only the conditional variances in the numerator of (19) affect the ordering (according to their significance) of the input variables. In this way, it is possible to identify the least significant variables at a small computational cost. These variables can be safely assigned to lower polynomial degrees, or even be treated as deterministic, thus reducing the cardinality of the basis set.

\section{Numerical Results}

We first examine a periodic-grating optical structure [27], which is designed to produced flat, high-efficiency and broad reflection spectra. The geometry of the considered filter is shown in Fig. 2, and its nominal geometric parameters are: $f_{1}=f_{3}=0.283, f_{2}=1, \Lambda=846.4 \mathrm{~nm}$, $d_{1}=d_{3}=375 \mathrm{~nm}$, and $d_{2}=175 \mathrm{~nm}$ [27]. We are interested in investigating the properties of the reflectance for normal incidence, as a function of the wavelength, when the aforementioned variables are treated as stochastic. Specifically, this problem features 7 geometric random inputs with

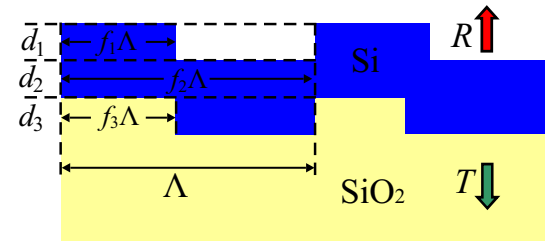

Figure 2: Geometry of the periodic-grating optical filter, depicting the geometric parameters that are considered stochastic.

uniform distributions, in the range within $\pm 1 \%$ of the corresponding mean (nominal) values. The computation of the structure's reflectance is performed with the Rigorous Coupled Wave Analysis [28], a frequency-domain convergent solver that has become a standard tool for problems with periodic-grating configurations. As described earlier, the proposed methodology features a two-stage implementation. For the initial step, the 1-D basis functions of a totaldegree PC expansion with $p=4$ are selected, whose overall number is $4 \times 7+1=29$ basis functions. By performing only 27 simulations and implementing CS, an estimation of the Sobol indices at every wavelength is derived, based on which the selection of the three most important variables is carried out. Next, a new, total-degree truncation set is constructed, considering only the dominant factors. The new set comprises 35 basis functions, and the corresponding coefficients are recovered by CS and another 27 deterministic samples. Consequently, a total of only $27+27=54$ simulations are performed.

Fig. 3 plots the mean value and the standard deviation of the reflectance, when computed with a standard MC approach and two CS methodologies. The MC curves are obtained after 10,000 simulations and used as the reference data. The comparison clearly verifies that the sparse solutions provide credible statistics for the QoI, despite using only a small number of deterministic samples. The results also prove that, for the considered level of uncertainty, the flatness of the reflectance is maintained to a high degree, and the most notable variability is noted at the two ends of the spectrum, where the transition of the reflectance between high and low values takes place. In addition, we compute the PDF of the reflectance at the wavelength where the highest standard deviation is observed (around 1.328 $\mu \mathrm{m})$. The comparison of the different curves (computed via kernel-density estimation [29]) is shown in Fig. 4, and good agreement of the sparse models with the reference solution can be noted again.

A dielectric-stack filter is examined next, which consists of 10 lossless slabs, each one with a mean relative permittivity $\epsilon_{r}=3.5$, and width equal to $1.2 \mathrm{~cm}$ (Fig. 5). The slabs are placed apart, at a distance of $3.6 \mathrm{~cm}$. We examine the transmission coefficient upon normal incidence, when the $\epsilon_{r}$ of each slab varies within $\pm 10 \%$ of the mean value. Now, the deterministic solver is based on the finitedifference time-domain method [30]. We select $p=3$ for 


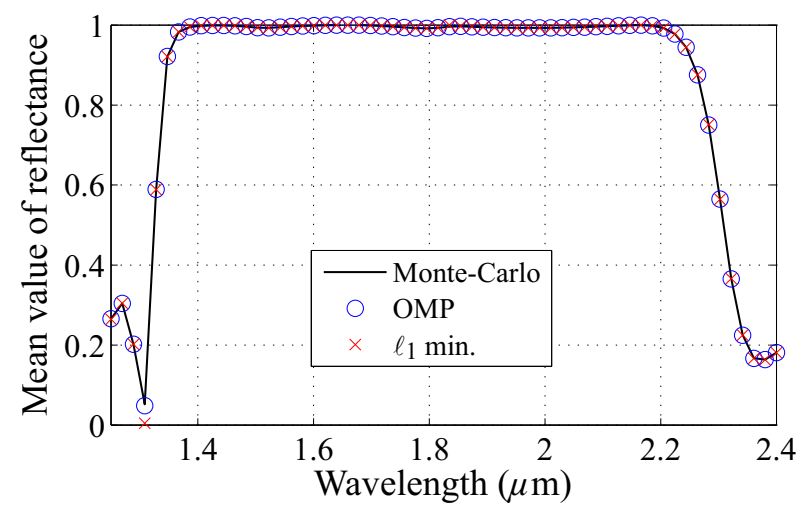

(a)

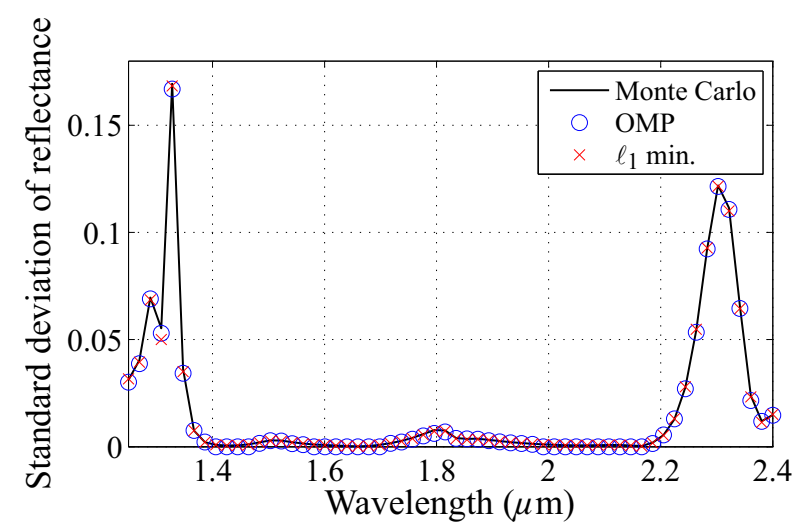

(b)

Figure 3: (a) Mean value and (b) standard deviation of the reflectance of the periodic-grating optical filter of Fig. 2 for normal incidence.

the initial basis set, which comprises 286 functions. The first step of the proposed procedure enables the computation of the partial variances of all variables throughout the considered frequency band. This action requires a basis set with only $3 \times 10+1=31$ functions. Unlike the first problem, we implement a least-squares approach, and then average the partial variances over all frequencies. In this way, we determine each variable's importance considering the average values, and the same reduced basis set is used at every frequency point. In fact, we choose to construct an anisotropic basis set (instead of simply rejecting the weaker variables), where the order $p$ is set to 3 for the 4 most important variables, to 2 for other 4 variables, and to only 1 for the least influential factors. The resulting basis set comprises 67 elements, and the amount of random samples is selected equal to 50 for both the OMP and the $\ell_{1}$-minimization approaches.

Before proceeding with some numerical results for the configuration of Fig. 5, we pay attention to the reliability of the first stage of the proposed technique. Table I compares the average partial variances, when obtained via full total-degree PC expansions with $p=3$, or the suggested line of work. In essence, the former are used as a reference for assessing the reliability of the latter. As the approxima-

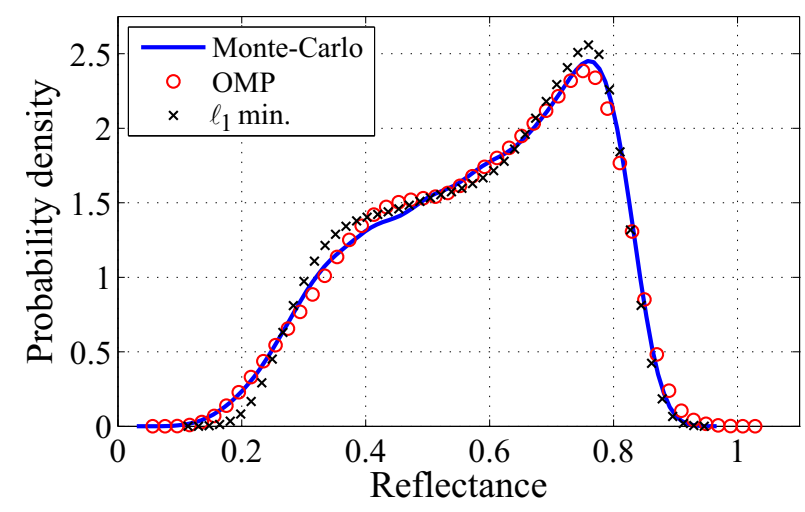

Figure 4: Probability-density function of the reflectance at the wavelength of maximum variance (approximately 1.328 $\mu \mathrm{m})$.

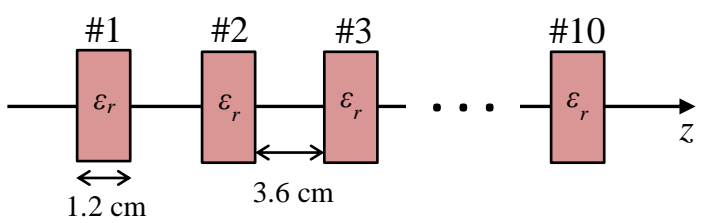

Figure 5: Geometry of the dielectric-stack filter, comprising 10 slabs with stochastic electric permittivities.

tion of the partial variances is conducted using a basis set of 31 basis functions, 62 simulations are required for the calculation of the expansion coefficients, in the context of a least-squares approach. For this problem, we compute the partial variance $\operatorname{var}_{i}(f)$ for the $i$-th variable, $i=1, \ldots, 10$ as a function of frequency, and then obtain the corresponding averages over all frequencies $f \in\left[f_{\min } f_{\max }\right]$, via

$$
\left\langle\operatorname{var}_{i}\right\rangle=\frac{1}{f_{\text {max }}-f_{\min }} \int_{f_{\text {min }}}^{f_{\text {max }}} \operatorname{var}_{i}(f) d f
$$

As the comparison in Table I verifies, the computation of the partial variances with the reduced basis set can be considered reliable, in the sense that it can be used for ordering the random variables according to their significance, despite some discrepancies with the actual values, obtained with the full PC expansion.

The mean value and the standard deviation of the transmission coefficient $T$ are shown in Fig. 6, where satisfactory matching with the reference data, based on $30,000 \mathrm{MC}$ simulations, is evident. This accuracy comes at a much lower computational cost, since the derivation of the sparse solution requires $50+62=112$ simulations, whereas the full PC expansion necessitates at least $2 \times 286=572$ samples. It can be noted that, similarly to the previous problem, the highest variability is observed at the transition area of the transmission coefficient, while the flatness is maintained in the band $2.2-3 \mathrm{GHz}$, as practically no variability is encountered therein. An illustration of the PC coefficients, computed by the OMP algorithm and the $\ell_{1}$ - 
Table 1: Estimated average partial variances $(\times 1000)$ for the stack-dielectric problem.

\begin{tabular}{|c|c|c|c|}
\hline Significance & Variable & Full PC & PC (1D bases) \\
\hline 1 & $\epsilon_{r 6}$ & 5.42 & 6.76 \\
\hline 2 & $\epsilon_{r 5}$ & 5.37 & 6.04 \\
\hline 3 & $\epsilon_{r 4}$ & 3.61 & 4.37 \\
\hline 4 & $\epsilon_{r 7}$ & 3.57 & 4.17 \\
\hline 5 & $\epsilon_{r 3}$ & 2.75 & 3.58 \\
\hline 6 & $\epsilon_{r 8}$ & 2.74 & 3.24 \\
\hline 7 & $\epsilon_{r 9}$ & 1.65 & 2.10 \\
\hline 8 & $\epsilon_{r 2}$ & 1.64 & 1.62 \\
\hline 9 & $\epsilon_{r 10}$ & 0.35 & 0.57 \\
\hline 10 & $\epsilon_{r 1}$ & 0.35 & 0.33 \\
\hline
\end{tabular}

minimization approach, is given in Fig. 7. It can be deduced that the sparse solutions maintain, to a satisfactory degree, the most important elements of the total-degree PC expansion, and neglect several terms of low significance at the same time. It can be also noted that the OMP solution is sparser than the corresponding one of the $\ell_{1}$-minimization technique.

We also calculate the distribution of the 6-dB roll-off frequency, whose value is affected by the uncertainty in the electric parameters of the stack filter (Fig. 8 depicts a fraction of different transmission-coefficient curves, computed by the MC method, and illustrates this phenomenon). A comparison of the PDFs computed via MC, OMP, and $\ell_{1}$-minimization is depicted in Fig. 9. Although some deviations may be observed, compared to the results of the previous example (probably due to the higher dimensionality and stronger input variability), it appears that the sparse polynomial surrogates correctly incorporate the most fundamental statistical properties of the physical QoI. Thus, they can be safely utilized, without resorting to alternatives with higher computational cost. It is interesting to notice that the considered levels of uncertainty in the values of the dielectric constants results in randomness in the 6-dB rolloff frequencies that span a band of almost $100 \mathrm{MHz}$.

\section{Conclusion}

This paper has focused on developing sparse PC models for the investigation of stochastic problems involving EM filters with various uncertain parameters. The implementation of known CS solvers has been further facilitated by a variance analysis at an early stage, which provides sufficient information regarding the influence of each input and enables a more consistent choice of the basis set, unlike standard methodologies. A number of numerical ex-

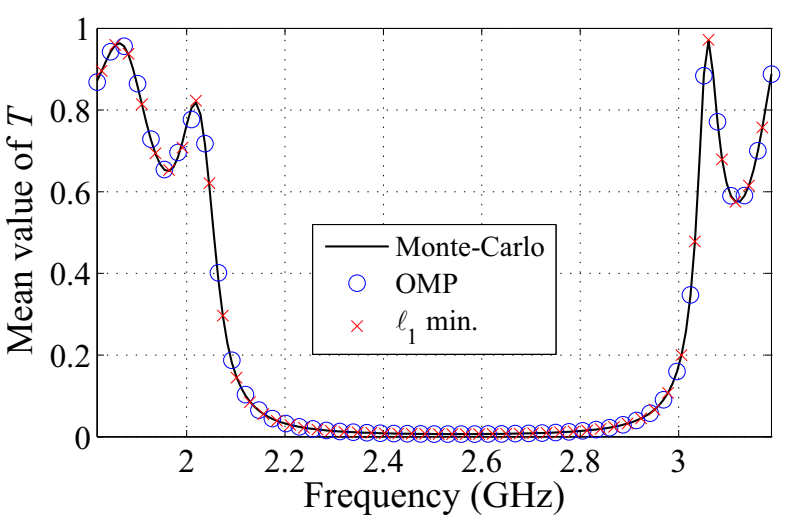

(a)

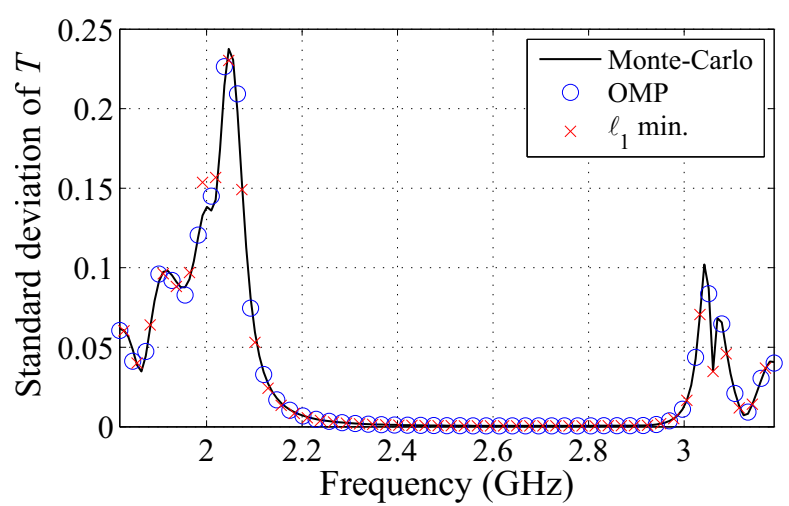

(b)

Figure 6: (a) Mean value and (b) standard deviation of the transmission coefficient $T$ of the dielectric-stack filter of Fig. 5 for normal incidence.

periments have verified the validity of the proposed line of work and exemplified its efficiency. Finally, the impact of uncertainty on the performance of the examined filtering structures has been assessed, and it has been deduced than non-trivial variability may emerge, even in the presence of weak uncertainties.

\section{Acknowledgement}

A. D. Papadopoulos acknowledges support from the framework of the EUROfusion Consortium and has received funding from the Euratom research and training programme 2014-2018 and 2019-2020 under grant agreement No 633053. The views and opinions expressed herein do not necessarily reflect those of the European Commission.

\section{References}

[1] A. Tombak, J. . Maria, F. T. Ayguavives, Zhang Jin, G. T. Stauf, A. I. Kingon, and A. Mortazawi, "Voltage-controlled RF filters employing thin-film barium-strontium-titanate tunable capacitors," IEEE Trans. Microw. Theory Tech., vol. 51, no. 2, pp. 462467, Feb. 2003. 


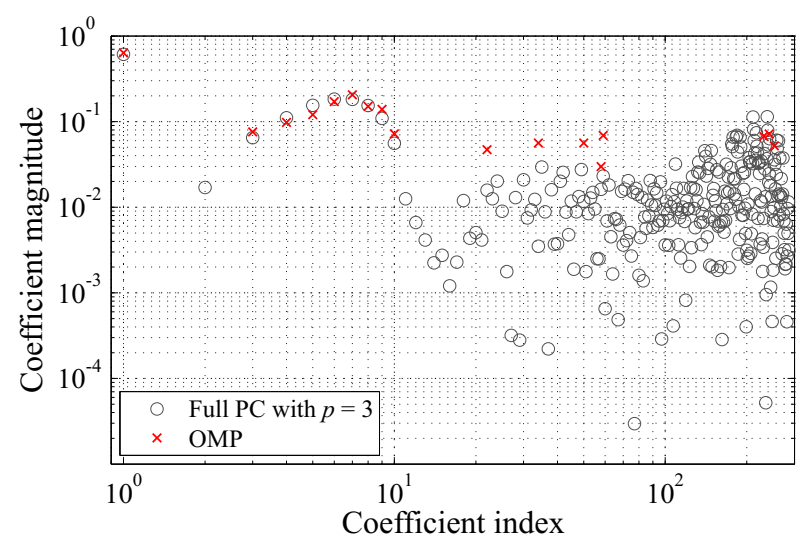

(a)

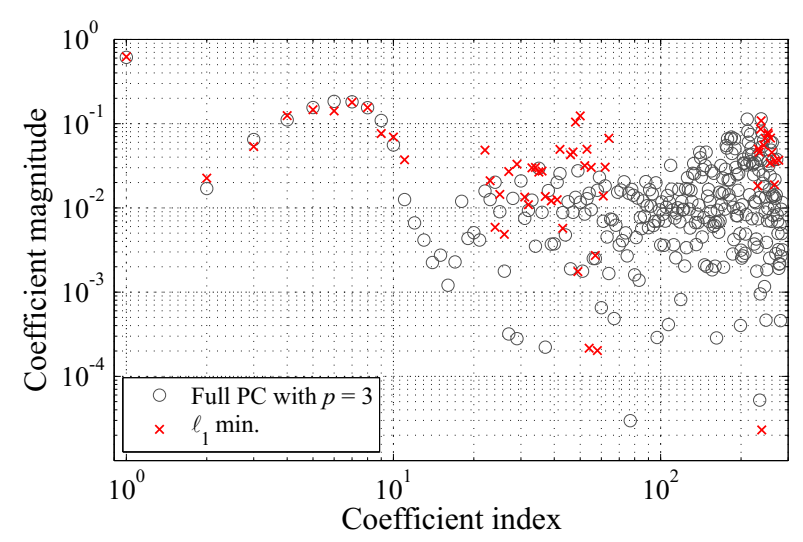

(b)

Figure 7: Magnitude of PC coefficients obtained via (a) OMP and (b) $\ell_{1}$-minimization.

[2] D. Swanson and G. Macchiarella, "Microwave filter design by synthesis and optimization," IEEE Microw. Mag., vol. 8, no. 2, pp. 55-69, Apr. 2007.

[3] A. Kumar, R. Patel, and M. V. Kartikeyan, "Investigation on microstrip filters with CSRR defected ground structure," AEM, vol. 5, no. 2, pp. 28-33, Sept. 2016.

[4] P. Blondy, A. R. Brown, D. Cros, and G. M. Rebeiz, "Low-loss micromachined filters for millimeter-wave communication systems," IEEE Trans. Microw. Theory Tech., vol. 46, no. 12, pp. 2283-2288, Dec. 1998.

[5] I. H. Libon, S. Baumgrtner, M. Hempel, N. E. Hecker, J. Feldmann, M. Koch, and P. Dawson, "An optically controllable terahertz filter," Appl. Phys. Lett., vol. 76, no. 20, pp. 2821-2823, 2000.

[6] R. Magnusson and S. S. Wang, "New principle for optical filters," Appl. Phys. Lett., vol. 61, no. 9, pp. 1022-1024, 1992.

[7] M. Swillam, Y. Ismail, and R. Kotb, "Tunable nanoplasmonics," AEM, vol. 2, no. 2, pp. 1-5, July 2013.

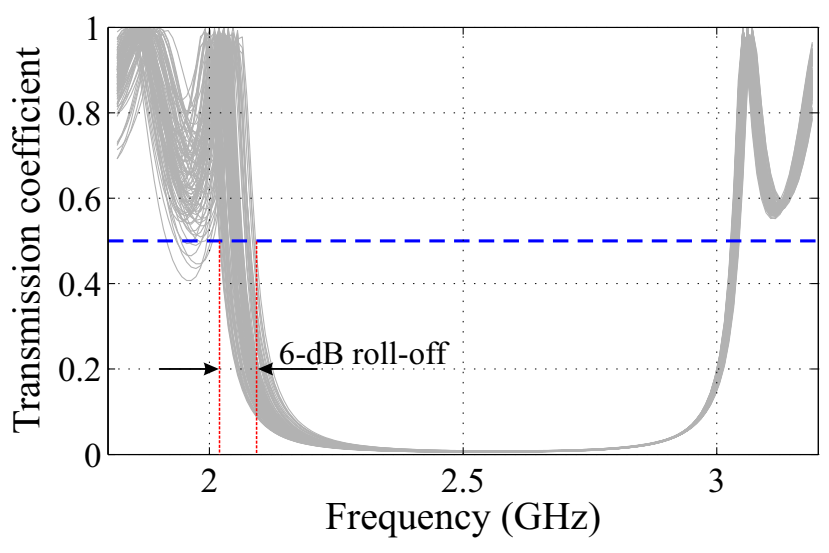

Figure 8: Uncertainty in the 6-dB roll-off frequency (100 MC curves are depicted).

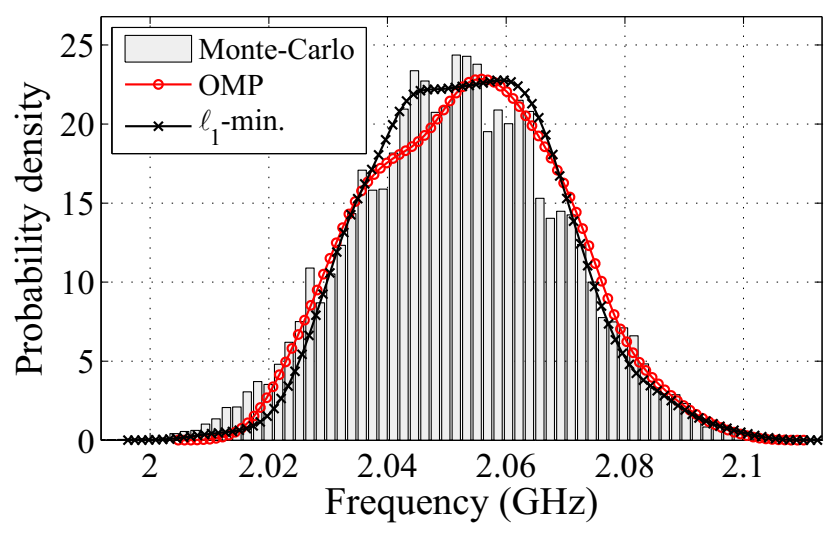

Figure 9: Probability-density functions of the 6-dB roll-off frequencies, in the case of the dielectric-stack filter.

[8] D. Xiu and G. Karniadakis, "The Wiener-Askey polynomial chaos for stochastic differential equations," SIAM J. Sci. Comput., vol. 24, no. 2, pp. 619-644, 2002.

[9] C. P. Robert, Monte Carlo Methods. John Wiley \& Sons, Ltd, 2014.

[10] D. Xiu and J. Hesthaven, "High-order collocation methods for differential equations with random inputs," SIAM J. Sci. Comput., vol. 27, no. 3, pp. 11181139, 2005.

[11] G. Blatman, "Adaptive sparse polynomial chaos expansions for uncertainty propagation and sensitivity analysis," Ph.D. dissertation, 2009, thése de doctorat dirigée par Sudret, Bruno Génie mécanique ClermontFerrand 22009.

[12] G. Blatman and B. Sudret, "Adaptive sparse polynomial chaos expansion based on least angle regression," J. Comput. Phys., vol. 230, no. 6, pp. 2345 - 2367, 2011. 
[13] L. Yan, L. Guo, and D. Xiu, "Stochastic collocation algorithms using $\ell_{1}$-minimization," Int. J. Uncertain. Quan., vol. 2, no. 3, pp. 279-293, 2012.

[14] J. Peng, J. Hampton, and A. Doostan, "A weighted $\ell_{1}$ minimization approach for sparse polynomial chaos expansions," J. Comput. Phys., vol. 267, pp. 92-111, 2014.

[15] J. Hampton and A. Doostan, "Compressive sampling of polynomial chaos expansions: Convergence analysis and sampling strategies," J. Comput. Phys., vol. 280, pp. $363-386,2015$.

[16] J. Jakeman, M. Eldred, and K. Sargsyan, "Enhancing $\ell_{1}$-minimization estimates of polynomial chaos expansions using basis selection," J. Comput. Phys., vol. 289 , pp. $18-34,2015$.

[17] D. Loukrezis, "Adaptive approximations for highdimensional uncertainty quantification in stochastic parametric electromagnetic field simulations," Ph.D. dissertation, Technische Universität, Darmstadt, Feb. 2019.

[18] X. Zhu and B. Sudret, "Replication-based emulation of the response distribution of stochastic simulators using generalized lambda distributions," Nov. 2019. [Online]. Available: https://hal.archivesouvertes.fr/hal-02373464

[19] A. Alexanderian, P. A. Gremaud, and R. C. Smith, "Variance-based sensitivity analysis for timedependent processes," Reliab. Eng. Syst. Safe., p. 106722, 2019.

[20] S. Wu, A. Dimakis, S. Sanghavi, F. Yu, D. HoltmannRice, D. Storcheus, A. Rostamizadeh, and S. Kumar, "Learning a compressed sensing measurement matrix via gradient unrolling," in Proceedings of the 36th International Conference on Machine Learning, ser. Proceedings of Machine Learning Research, K. Chaudhuri and R. Salakhutdinov, Eds., vol. 97. Long Beach, California, USA: PMLR, 09-15 June 2019, pp. 6828-6839.

[21] A. Tarakanov and A. H. Elsheikh, "Regression-based sparse polynomial chaos for uncertainty quantification of subsurface flow models," J. Comput. Phys., vol. 399, p. 108909, 2019.

[22] I. Kapse, A. K. Prasad, and S. Roy, "Generalized anisotropic polynomial chaos approach for expedited statistical analysis of nonlinear radio-frequency (RF) circuits," in 2016 IEEE 20th Workshop on Signal and Power Integrity (SPI), May 2016, pp. 1-3.

[23] E. van den Berg and M. P. Friedlander, "SPGL1: A solver for large-scale sparse reconstruction," http://www.cs.ubc.ca/labs/scl/spgl1, June 2007.
[24] E. van den Berg and M. Friedlander, "Probing the pareto frontier for basis pursuit solutions," SIAM J. Sci. Comput., vol. 31, no. 2, pp. 890-912, 2009.

[25] N. Alemazkoor and H. Meidani, "A near-optimal sampling strategy for sparse recovery of polynomial chaos expansions," J. Comput. Phys., vol. 371, pp. 137 $151,2018$.

[26] G. Blatman and B. Sudret, "Anisotropic parcimonious polynomial chaos expansions based on the sparsityof-effects principle,' in Proc ICOSSAR'09, International Conference in Structural Safety and Reliability, 2009.

[27] M. Shokooh-Saremi and R. Magnusson, "Leakymode resonant reflectors with extreme bandwidths," Opt. Lett., vol. 35, no. 8, pp. 1121-1123, Apr. 2010.

[28] M. G. Moharam, E. B. Grann, D. A. Pommet, and T. K. Gaylord, "Formulation for stable and efficient implementation of the rigorous coupled-wave analysis of binary gratings," J. Opt. Soc. Am. A, vol. 12, no. 5, pp. 1068-1076, May 1995.

[29] B. W. Silverman, Density Estimation for Statistics and Data Analysis. London: Chapman \& Hall, 1986.

[30] A. Taflove and S. C. Hagness, Computational electrodynamics: The finite-difference time-domain method. Norwood MA: Artech House, 2005. 